\title{
Well log data super-resolution based on locally linear embedding
}

\author{
Jian Han (1), Pan Gao (D), Zhimin Cao* (D), Jing Li (10, Sijie Wang (D), and Can Yang \\ College of Physics and Electronic Engineering, Northeast Petroleum University, Daqing 163318, PR China
}

Received: 7 February 2021 / Accepted: 21 July 2021

\begin{abstract}
Unconventional remaining oil and gas resources such as tight oil, shale oil, and coalbed gas are currently the focus of the exploration and development of major oil fields all over the world. Therefore, to make best understand of target reservoirs, enhancing the vertical resolution of well log data is crucial important. However, in the face of the continuous low-level fluctuations of international oil price, large scale use of expensive high resolution well logging hardware tools has always been unaffordable and unacceptable. In another aspect, traditional well log interpolation methods can always not realize high reliable information enhancement for crucial high frequency components. In this paper, in order to improve the well log data super-resolution performance, we propose for the first time to employ Locally Linear Embedding (LLE) technique to reveal the nonlinear mapping relationship between 2-times-scale-difference well log data. Several super resolution experiments with well log data from a given area of Daqing Oil field, China, were conducted. Experimental results illustrated that the proposed LLE-based method can efficiently achieve more reliable super-resolution results than other state-of-the-art methods.
\end{abstract}

\section{Introduction}

In recent years, most old oilfields in the world have entered the middle and late stages of development. However, due to the long-term waterflooding development of oilfields, several unavoidable complex cases happened to the conventional resources, such as frequent river channel reconstruction, complex vertical and horizontal changes of sand body, strong heterogeneity of lithology and lithofacies. Consequently, oil and gas exploration and development are becoming more and more difficult, and unconventional oil and gas resources such as tight sandstone, shale oil/gas, coalbed gas, etc, have become the most important target reservoirs. Obviously, the efficient exploration and development of unconventional oil and gas resources has become the main way to achieve stable production to increase and extend the life of oilfield development [1]. Therefore, in order to enhance the production capacity of the unconventional remaining oil and gas resources, the reservoir modelling resolution should be as high as possible. Meanwhile, the high-resolution logging curves can better provide the possibility to predict the location of lost circulation [2], lithology identifies [3], and predict the productivity of heterogeneous reservoirs $[4,5]$. In practice, because the vertical resolution of seismic data is too low and the cost of acquisition of core and other high resolution logging data is too high, it is an inevitable choice to employ conventional

\footnotetext{
* Corresponding author: 2645073549@qq.com
}

well log data to enhance the vertical resolution of the final reservoir models. However, in the conventional oil/gas era, interpolation algorithms based on simple models have been used for enhancing the vertical resolution of well log data for a very long time. Until the last decade or so, with the development of machine learning technology, a few data driven well log super-resolution methods have been proposed in the literature. Furthermore, whether it is a simple model-driven interpolation method or a data-driven superresolution method, they all try to establish a non-linear mapping function between low resolution and highresolution data directly in the original amplitude space. Due to some uncontrollable factors, there are unavoidable data deficiencies existing in the raw well data, therefore, the quality of training data severely restricts the effectiveness and robustness of these super-resolution methods. In order to deal with this problem to a certain extent, Locally Linear Embedding (LLE) technique which can explore intrinsic manifold of the raw data with sufficient outlierresistance and robustness was employed in this paper to perform well super-resolution task. Therefore, in this paper, a novel LLE based well logs super resolution method was proposed in the literature for the first time.

\section{Related works}

In the actual survey work of the oil field, due to the influence of factors such as well diameter and measurement, 
the actual logging resolution that can be achieved at present is only $12.5 \mathrm{~cm}$. Faced with complex geological conditions, the thickness of shale oil lamellae is less than $0.3 \mathrm{~cm}$. Therefore, it is not realistic to accurately characterize the target reservoir using original logging data. Obviously, in order to enhance capacity of reservoir characterization accuracy, the resolution of well log data must be improved. However, due to the high cost of high-resolution well logging tools, a series of methods to improve the logging resolution was proposed in the literature. In 1989, Flaum et al. [6] proposed the neutron density $\alpha$ factor method. By assuming that the $\alpha$ factor changes slowly, the high-resolution neutron porosity curve can be calculated by using the counting rate of near detector. In 1991, Nelson, and Mitchell [7] deduced the expression of smoothing filter used for curve matching and proposed the resolution matching technology for high resolution processing of well log data. On this basis, [8] used genetic algorithm to enhance the resolution of well log data. Specifically, they first used genetic algorithm to determine the filter in the frequency domain and then used resolution matching technology to improve the resolution of the input well $\log$ data. Conaway [9] discussed the deconvolution technology of natural gamma ray data with point detector. Based on the three-point deconvolution formula, and proposed a method for determining the shape constant $\alpha$, which appropriately considers the influence of formation dip. Freedman and Minerbo [10] employed the maximum entropy deconvolution method to improve the vertical resolution of well log data. By taking the layer interface information into consideration, they formulated a Lagrange optimal function with maximum entropy constraint for super resolution, and reasonable vertical resolution enhancement can be achieved. Besides to the method processed in spatial domain, transformed domain based well $\log$ super-resolution methods can achieve relative better performance. In 2005, Tai and Cao [11] used Walsh transform to improve the resolution of well $\log$ data. Furthermore, their method can also ensure that the error between the calculated value and the true value does not exceed the error of the raw well log data itself. However, this method is only suitable for linear response well log data and the response function $H(\tau)$ cannot be accurately determined. In 2015, Li et al. [12] proposed to employ window Fourier transform to transform the raw well log data from the spatial domain to the frequency domain. The relationship between high-resolution and low-resolution well log data in the frequency domain can therefore be constructed.

Although the above-mentioned model-driven methods can improve the well log resolution to a certain extent, the original outliers or errors in the raw well log data will be certainly propagated or even enlarged to the corresponding high-resolution data. To deal with similar problems, in the computer audio/vision fields, sparse representation and deep learning techniques have been successfully applied to the image super-resolution task [13-17]. In 2017, Ledig et al. [18] proposed an image super-resolution method based on generative adversarial network. Also in 2017, Volodymyr et al. [19] proposed a multi-layer convolution neural network for high-resolution audio signal processing.
In 2018, Lim et al. [20] proposed a novel deep neural network structure to perform audio super-resolution in time-frequency domain. Generally, promising superresolution results can be achieved in the computer audio/ vision fields by employing data-driven super-resolution techniques. However, compared with audio or image/video data, there are much more uncertainties existing in the well $\log$ data. Consequently, directly employing data-driven methods in spatial or classical transform domains cannot prevent the propagation of uncertainty to the superresolution version of data.

Based on the above review and analysis, we propose to employ manifold learning techniques, which can extract the intrinsic manifold of data with variable uncertainties, to enhance the super-resolution performance of well log data. Specifically, the LLE algorithm is used in this paper to exploit the intrinsic manifold information of well log data to conduct the corresponding super-resolution task.

\section{Locally Linear Embedding (LLE) based super-resolution}

\subsection{Locally Linear Embedding}

LLE algorithm is originally a nonlinear dimension reduction algorithm, which belongs to the category of manifold learning. Manifold learning is to recover intrinsic low dimensional structure from high-dimensional sampled data, that is, to find low dimensional manifold in highdimensional space and find the corresponding nonlinear mapping to achieve dimension reduction. Chang et al. [21] first proposed the use of LLE to deal with image superresolution and stable and promising results were illustrated. Specifically, for the LLE algorithm, suppose there are $m$ $n$-dimensional samples $\left\{\boldsymbol{X}_{1}, \boldsymbol{X}_{2}, \boldsymbol{X}_{3}, \cdots \boldsymbol{X}_{\mathrm{m}}\right\}$, the first step is to select the neighborhood size which is one of the hyperparameters used in the LLE algorithm. Without loss of generality, assuming that the neighborhood size is $K$. Therefore, for a given data point $\boldsymbol{X}_{i}$, we assume that it can be represented by the weighted linear combination of its $K$ nearest neighbors $\boldsymbol{N}_{\boldsymbol{i}}=\left\{\boldsymbol{X}_{j}\right\}, j=1,2, \cdots, K$. And the corresponding mean square error is used as the loss function,

$$
J(w)=\sum_{i=1}^{m}\left\|X_{i}-\sum_{j=1}^{K} W_{i j} X_{j}\right\| .
$$

It should be noted that the weight coefficient $W_{i j}$ is local normalized (for any data $\boldsymbol{X}_{i} \notin N_{i}$, the corresponding weight coefficient is zero). It means that the sum of the weight coefficients corresponding to $\boldsymbol{X}_{i}$ 's neighborhood $\left\{\boldsymbol{X}_{j}\right\}, j=1$, $2, \cdots, K$ is 1 . Therefore, the weight coefficient should meet the following requirements:

$$
\sum_{i=1}^{K} W_{i j}=W_{i}^{T} 1_{k}=1 .
$$

With equation (2) as constraint, the weight coefficients in equation (1) can be obtained by Lagrange multiplier method as follows: 


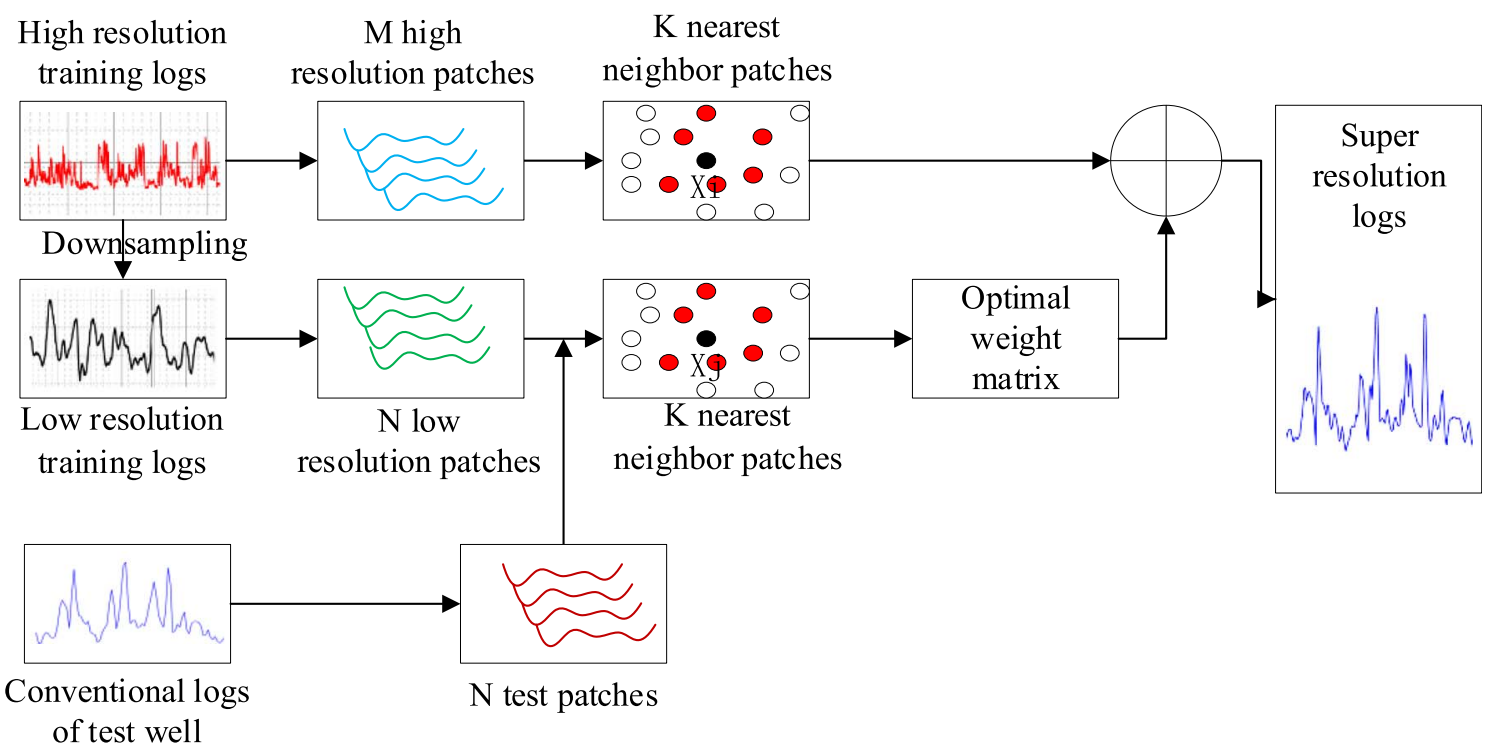

Fig. 1. Flow chart of locally linear embedding algorithm.

$$
\begin{aligned}
J(w) & =\sum_{i=1}^{m}\left\|X_{i}-\sum_{j=1}^{K} W_{i j} X_{j}\right\|_{2}^{2}=\sum_{i=1}^{m}\left\|\sum_{j=1}^{K} W_{i j} X_{j}-\sum_{j=1}^{K} W_{i j} X_{j}\right\|_{2}^{2} \\
& =\sum_{i=1}^{m}\left\|\sum_{j=1}^{K} W_{i j}\left(X_{i}-X_{j}\right)\right\|_{2}^{2}=\sum_{i=1}^{m}\left\|\left(X_{i}-X_{j}\right) W_{i}\right\|_{2}^{2} \\
& =\sum_{i=1}^{m} W_{i}^{T}\left(X_{i}-X_{j}\right)^{T}\left(X_{i}-X_{j}\right) W_{i} .
\end{aligned}
$$

Let $Z_{\mathrm{i}}=\left(X_{i}-X_{j}\right)^{T}\left(X_{i}-X_{j}\right)$, there will be:

$$
J(w)=\sum_{i=1}^{m} W_{i}^{T} Z_{\mathrm{i}} W_{i}
$$

Then, the optimization objective can be constructed as below:

$$
L(w)=\sum_{i=1}^{m} W_{i}^{T} Z_{\mathrm{i}} W_{i}+\lambda\left(W_{i}^{T} 1_{k}-1\right) .
$$

Next, setting the derivative of $L(W)$ to $W$ is 0 , the following results can be obtained:

$$
2 Z_{i} W_{i}+\lambda 1_{k}=0 .
$$

Let $\lambda^{\prime}=-\frac{1}{2} \lambda$ be a constant, then the final weight coefficient vector $W_{i}$ can be obtained as follows:

$$
W_{\mathrm{i}}=\frac{Z_{i}^{-1} 1_{k}}{1_{i}^{T} Z_{i}^{-1} 1_{k}}
$$

\subsection{LLE based well log data super-resolution}

From Section 3.1, we can see that LLE method can obtain the intrinsic relationship between a given $1 \times n$-dimensional local data patch and its $K$ nearest neighbor patches. Inspired by the Sparse Representation-based image SuperResolution (SRSR) method proposed in [12], in which low-resolution and high-resolution training data pairs were employed to share the same representation parameters. Therefore, combining the advantages of LLE and SRSR, two strategies were employed for well log super-resolution task. Firstly, let the low-resolution and high-resolution model data pairs share the same linear representation weights. Secondly, for the test data patches, let's find their $K$ nearest neighbors in the low-resolution model patches for LLE operation. With the guidance of these two strategies, the workflow diagram of the proposed well log superresolution method was illustrated in Figure 1.

Specifically, the detail of the proposed well log superresolution method was given in Table 1 .

\section{Experimental results}

\subsection{Geological background and data selection}

In order to evaluate the performance of the proposed well $\log$ super resolution method, well log data from 10 wells in Qijia-Gulong depression, Songliao basin, China were selected. Qijia-Gulong depression illustrated in Figure 2 is one of the most important tight sandstone exploration area of Daqing oil field. The average permeability of this area is commonly less than $1 \mathrm{mD}$, and the average porosity is always less than $13 \%$. Compared with these medium quality physical properties, the thickness of the target reservoir in this area is universally less than $3 \mathrm{~m}$, and there are always too many complex thin interlayers existed [22]. Specifically, without loss of generality, wells, natural Gamma Ray (GR), and Deep Lateral Resistivity (LLD) were selected for conducting the following experiments for all selected wells. 
Table 1. Illustration of the detailed steps of the proposed method.

Inputs: Model well $\log \boldsymbol{X}_{h}$, test low resolution $\log \boldsymbol{Y}_{l}$, local patch size $r$, sampling interval $\delta$, and neighborhood size $K$.

Step 1: Constructing model data pairs $\left(\boldsymbol{M}_{l}, \boldsymbol{M}_{h}\right)$ :

Step 1.1: Down-sampling $\boldsymbol{X}_{h}$ to obtain the corresponding low resolution well $\log \boldsymbol{X}_{l}$;

Step 1.2: Using (low-resolution) patch size $r$ and (low-resolution) sampling interval being $\delta$ to continuously sample log patch pairs $\left(\boldsymbol{M}_{l}, \boldsymbol{M}_{h}\right)$ from $\boldsymbol{X}_{l}$ and $\boldsymbol{X}_{h}$;

Step 2: Initialing the expected super-resolution output $\boldsymbol{Y}_{h}$ and the corresponding counting vector $\boldsymbol{C}_{h}$ to zeros.

Step 3: Conducting LLE-based super-resolution operation to $\boldsymbol{Y}_{l}$ :

For $k=1: \delta$ : length $\left(\boldsymbol{Y}_{l}\right)$

Step 3.1: Obtaining the current local patch $\boldsymbol{Y}_{l}^{k}$;

Step 3.2: Finding the corresponding $K$ nearest neighbors from $\boldsymbol{M}_{i}$;

Step 3.3: Calculating the LLE-based weight coefficient vector $\boldsymbol{W}_{k}$ using equation (7);

Step 3.4: Estimating the corresponding super-resolution local patch $\boldsymbol{Y}_{h}^{k}$ using $\boldsymbol{W}_{k}$ and $\boldsymbol{M}_{h}$.

Step 3.5: Accumulating $\boldsymbol{Y}_{h}^{k}$ to $\boldsymbol{Y}_{h}^{\prime}$ 's corresponding indexes $\boldsymbol{I}_{h}^{k}$.

Step 3.6: $\boldsymbol{C}_{h}\left(\boldsymbol{I}_{h}^{k}\right)=\boldsymbol{C}_{h}\left(\boldsymbol{I}_{h}^{k}\right)+1$;

\section{End}

Step 4: Calculating the final $\boldsymbol{Y}_{h}$ :

$$
\boldsymbol{Y}_{h}=\boldsymbol{Y}_{h} / \boldsymbol{C}_{h}(8)
$$

Outputs: High resolution
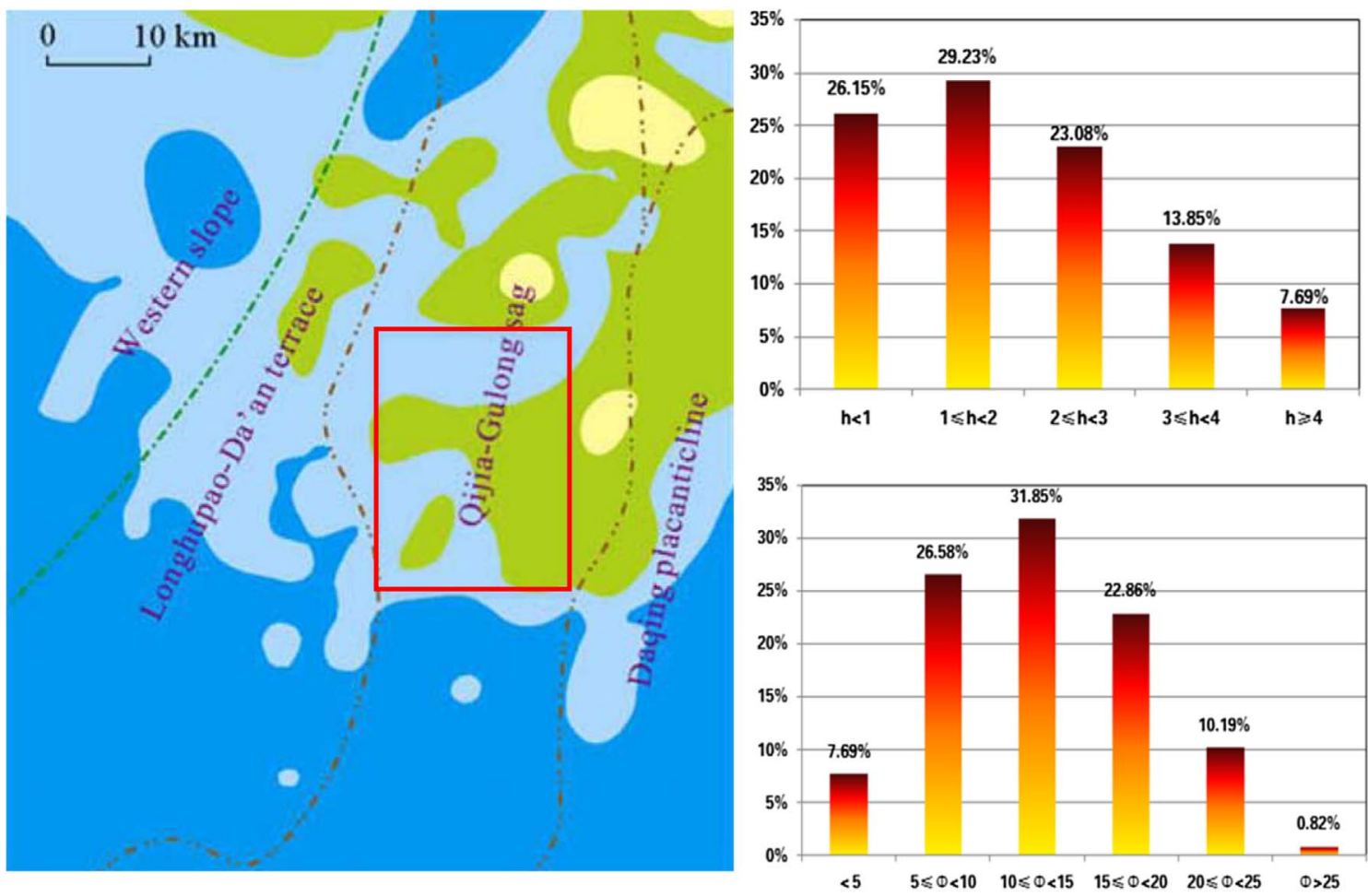

Fig. 2. Illustration of the geological background of the selected well data: the basic phase map of the Qijia-Gulong depression (left), the histogram of the thickness of sand body (right-up), and the histogram of the porosity (right-bottom).

\subsection{Hyper-parameter settings}

As mentioned in Section 3, the local patch size and the number of neighborhood patches are important hyper parameters of the method. Therefore, in this experiment, the influence of these two parameters on super-resolution results was discussed. Specifically, without loss of generality, we chose the GR curve as the test data. Firstly, using the same local patch size $(r=4)$, the performance of the proposed method was tested with neighborhood size 


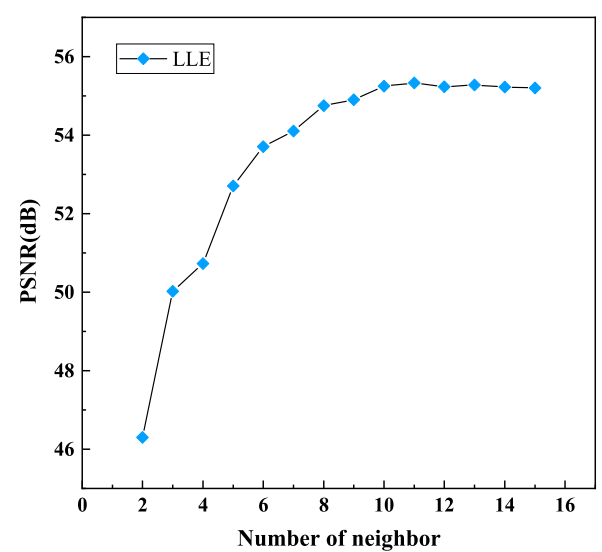

(a)

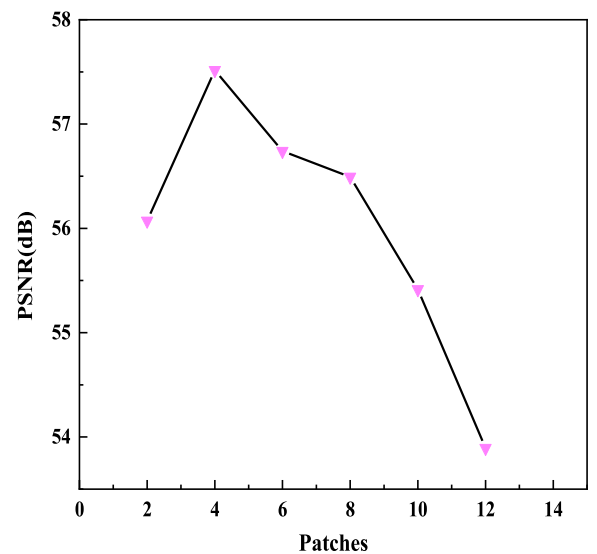

(b)

Fig. 3. Comparison of the super-resolution performance of the proposed method under a) different neighborhood sizes and b) different local patch sizes.

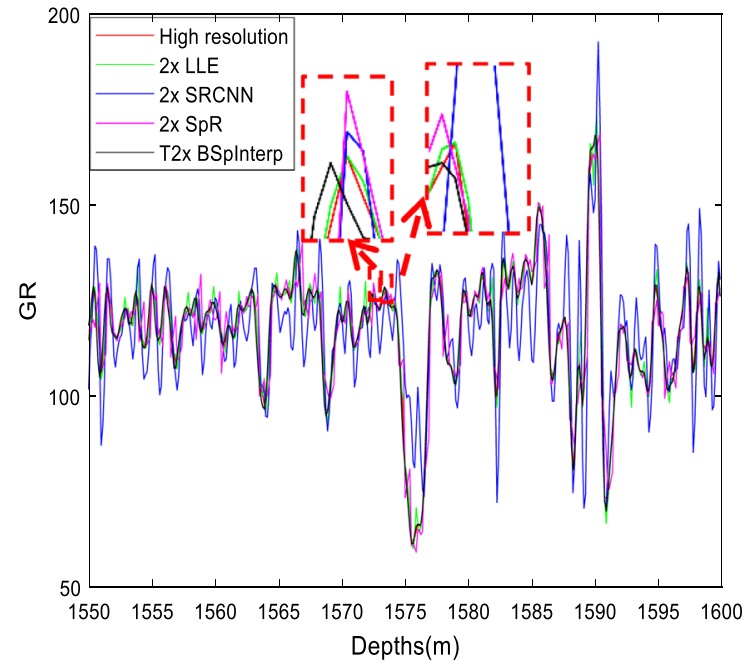

(a)

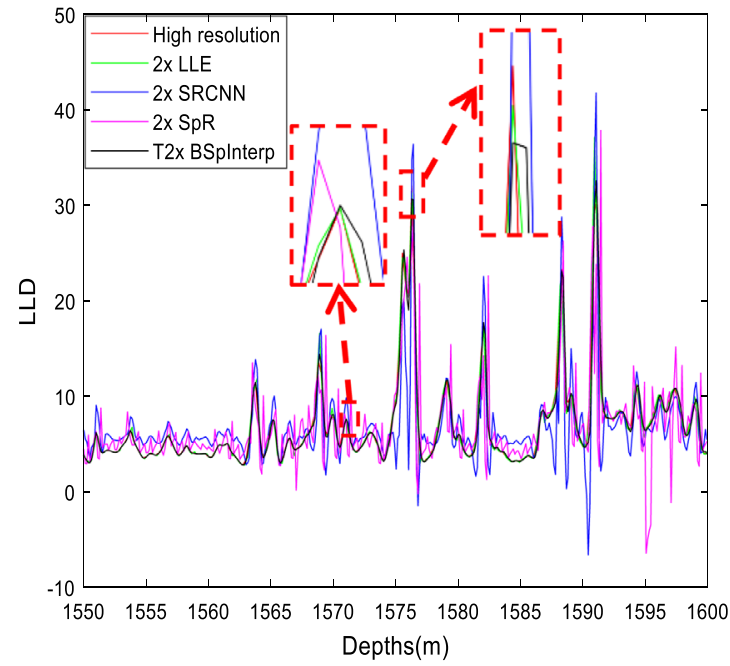

(b)

Fig. 4. Illustration of $2 X$ super-resolution results comparison of different methods for a) GR curve and b) LLD curve.

varying from 2 to 16 . As can be seen from Figure 3a, as the neighborhood size increases, the Peak Signal-to-Noise Ratio (PSNR) value improves rapidly, and when $K$ is set to 10 , the result tends to be stable. In addition, it is obvious that when $K$ is 11 , the best super-resolution result can be achieved. Meanwhile, by setting $K$ to 11 , as shown in Figure $3 \mathrm{~b}$, with the increase of the local patch size, the best super-resolution result is found when $r$ is set to 4 .

\subsection{Super resolution performance test}

In this experiment, the standard $0.125 \mathrm{~m}$ GR and LLD logging data from $1550 \mathrm{~m}$ to $1600 \mathrm{~m}$ section of well_1 was selected as the test data, and the corresponding section of well_2 was selected as model data. The corresponding $0.25 \mathrm{~m}$ low resolution curve is obtained by down-sampling. In order to verify the superiority of this method, the Bicubic Spline Interpolation method (BSpInterp for short), onedimensional Convolutional Neural Network (SRCNN for short) method, and the Sparse Representation method (SpR for short) were used for comparison.

Specifically, the comparison was conducted in three manners: (1) direct two times $(2 X)$ super-resolution; (2) double two times (indirect $4 X$ ) super-resolution; and (3) direct four times $(4 X)$ super-resolution. Then, as discussed in Section 4.2, local patch size and the neighborhood size used in the proposed method were setting to 4 and 11, respectively. Experimental results of different comparison manners were illustrated in Figure $4(2 X)$, Figure 5 (indirect $4 X$ ), and Figure $6(4 X)$, respectively.

In order to quantitatively evaluate the objective performance of these comparison methods, the Mean Square Error (MSE), Peak Signal-to-Noise Ratio (PSNR), and Pearson correlation Coefficient (Coeff) of the results acquired by each method were calculated. At the same time, the execution time required for each method is also recorded for comparison. Specifically, the detailed quantitative evaluation results of GR and LLD logging data were 


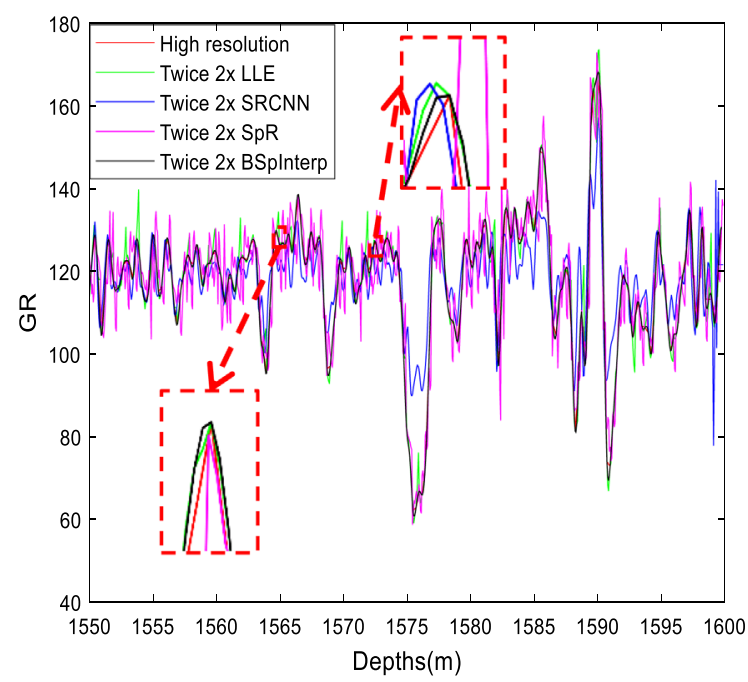

(a)

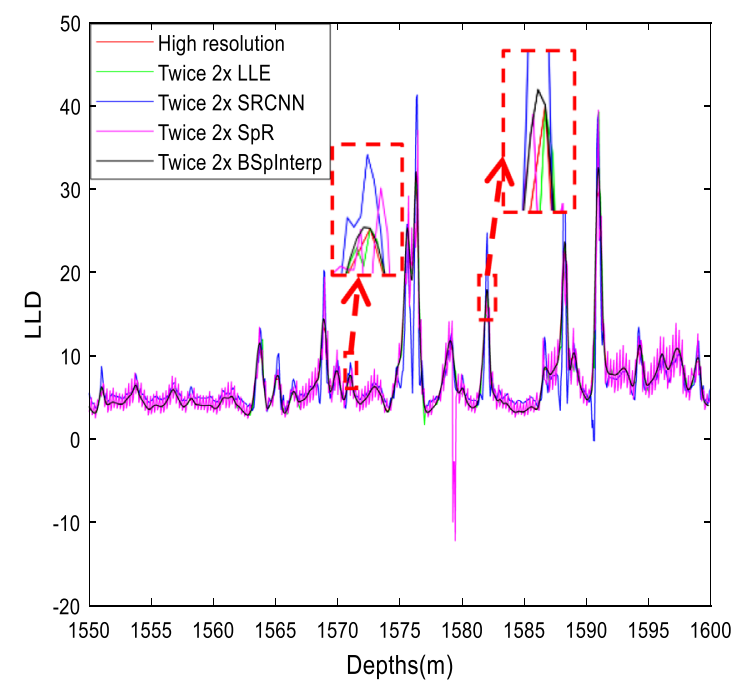

(b)

Fig. 5. Illustration of indirect $4 X$ super-resolution results comparison of different methods for a) GR curve and b) LLD curve.

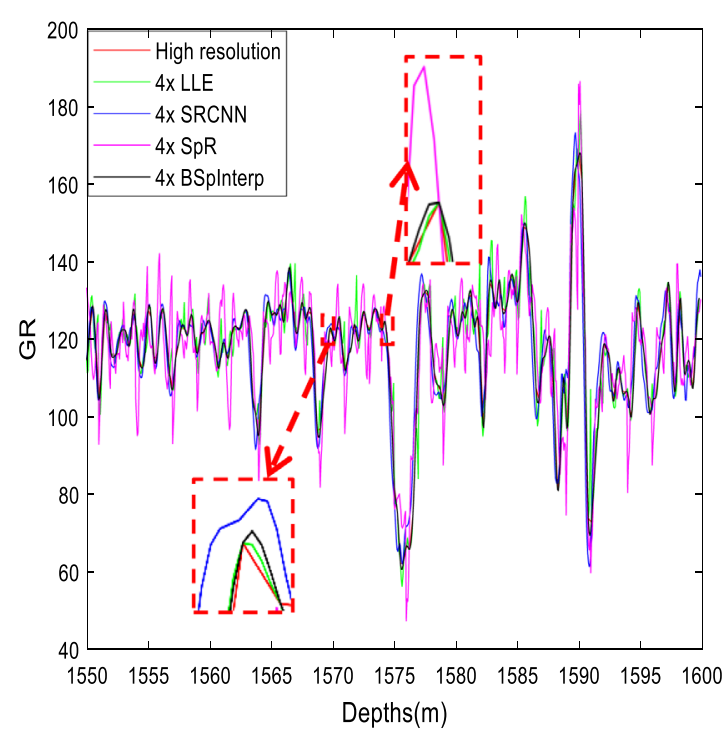

(a)

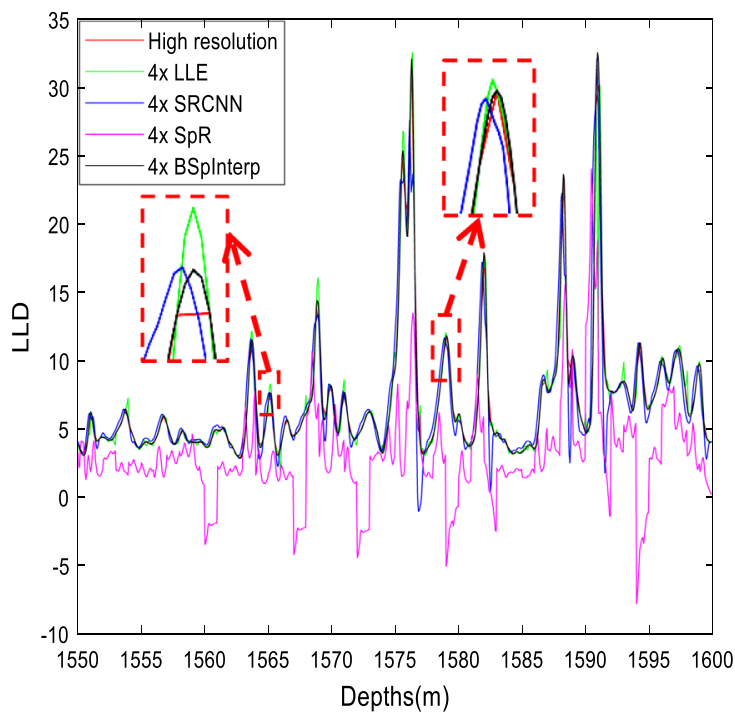

(b)

Fig. 6. Illustration of direct $4 X$ super-resolution results comparison of different methods for a) GR curve and b) LLD curve.

given in Tables 2 and 3, respectively. Our proposed method (LLE) achieves state-of-the-art results compared to other methods. The corresponding time-consuming data was given in Table 4.

\subsection{Super resolution comparison of logging data in different areas}

In order to verify the robustness of the proposed method, using well_2 as model well, the GR curves of the other eight wells were selected as test data using the $2 X$ superresolution manner. Specifically, for each well, $100 \mathrm{~m}$ log data was randomly extracted for processing. In this experiment, PSNR values of GR logs super-resolution results of different wells in different areas were compared. The detailed comparison data were given in Table 5. Our method has achieved the best results in 7 out of 8 oil wells. This result verifies the effectiveness of our method in different regions. Taking Well 3 as an example, the final superresolution results was shown in Figure 7 with lithology information for reference. Among them, the black curve is the original high-resolution curve, and the red curve is the super-resolution result.

\subsection{Discussion}

In order to evaluate the performance of the proposed well log super-resolution method, several experiments were 
Table 2. Comparison of quantitative evaluation results of different super-resolution methods for GR logging data.

\begin{tabular}{llcccc}
\hline & & BSpInterp & SpR & SRCNN & LLE \\
\hline \multirow{3}{*}{$2 X$} & MSE & 1.1292 & 5.8591 & 5.4859 & 0.0354 \\
& PSNR & 43.9911 & 36.8405 & 37.1264 & 68.0 \\
\multirow{3}{*}{ Indirect $4 X$} & Coeff & 0.2640 & 0.4291 & 0.7924 & 0.9946 \\
& MSE & 0.4454 & 6.3781 & 2.6682 & 0.0787 \\
& PSNR & 51.8654 & 32.5527 & 35.9087 & 64.5302 \\
Direct $4 X$ & Coeff & 0.7066 & 0.2590 & 0.6631 & 0.9005 \\
& MSE & 0.4454 & 6.2887 & 2.5339 & 0.0699 \\
& PSNR & 51.8654 & 33.3229 & 44.7912 & 65.0463 \\
& Coeff & 0.7066 & 0.5471 & 0.6638 & 0.9138 \\
\hline
\end{tabular}

Table 3. Comparison of quantitative evaluation results of different super-resolution methods for LLD logging data.

\begin{tabular}{llcccc}
\hline & & BSpInterp & SpR & SRCNN & LLE \\
\hline \multirow{3}{*}{$2 X$} & MSE & 0.3786 & 0.4409 & 0.3987 & 0.0687 \\
& PSNR & 39.3697 & 38.7085 & 39.1456 & 56.5083 \\
\multirow{3}{*}{ Indirect $4 X$} & Coeff & 0.2171 & 0.2198 & 0.7827 & 0.9147 \\
& MSE & 0.3791 & 3.1366 & 2.5099 & 0.0818 \\
& PSNR & 44.3598 & 35.5662 & 36.3842 & 54.0357 \\
Direct $4 X$ & Coeff & 0.4885 & 0.3098 & 0.6551 & 0.7322 \\
& MSE & 0.3791 & 3.9733 & 1.3088 & 0.0788 \\
& PSNR & 44.3598 & 30.6487 & 42.8876 & 54.0513 \\
& Coeff & 0.4885 & 0.2017 & 0.4418 & 0.7394 \\
\hline
\end{tabular}

Table 4. Time-consuming comparison of different super-resolution methods.

\begin{tabular}{llcccc}
\hline & & BSpInterp & SpR & SRCNN & LLE \\
\hline \multirow{3}{*}{$2 \mathrm{X}$} & GR & 0.42 & 1.37 & 1.20 & 0.08 \\
\multirow{3}{*}{ Indirect $4 \mathrm{X}$} & LLD & 0.39 & 1.30 & 1.18 & 0.06 \\
& GR & 1.44 & 3.19 & 5.43 & 1.26 \\
\multirow{2}{*}{ Direct $4 \mathrm{X}$} & LLD & 1.48 & 3.17 & 5.76 & 1.18 \\
& GR & 1.36 & 3.57 & 4.13 & 1.03 \\
& LLD & 1.28 & 3.38 & 4.24 & 0.98 \\
\hline
\end{tabular}

Table 5. Comparison of PSNR values of GR curves of different wells in different areas.

\begin{tabular}{lllllllll}
\hline PSNR & Well_3 & Well_4 & Well_5 & Well_6 & Well_7 & Well_8 & Well_9 & Well_10 \\
\hline BSpInterp & 55.7468 & 54.5416 & 56.7609 & 52.7531 & 53.7165 & 53.4290 & 55.4802 & 54.2892 \\
SpR & 42.0031 & 44.2984 & 43.6742 & 42.8289 & 42.4417 & 41.3376 & 43.2067 & 42.6666 \\
SRCNN & 37.0392 & 39.3897 & 42.6026 & 37.8745 & 37.4719 & 38.3754 & 38.2415 & 37.7311 \\
LLE & 55.7981 & 55.3966 & 57.9272 & 58.3226 & 53.0382 & 54.6592 & 57.4185 & 56.1093 \\
\hline
\end{tabular}

conducted. In Section 4.3, the direct visual effect and quantitative evaluation results of different methods were given. Specifically, from Figure 4, for $2 X$ super-resolution, we can see that SpR and SRCNN methods cannot effectively reconstruct the detailed information of well log, and there are relatively large fluctuations. In addition, the 


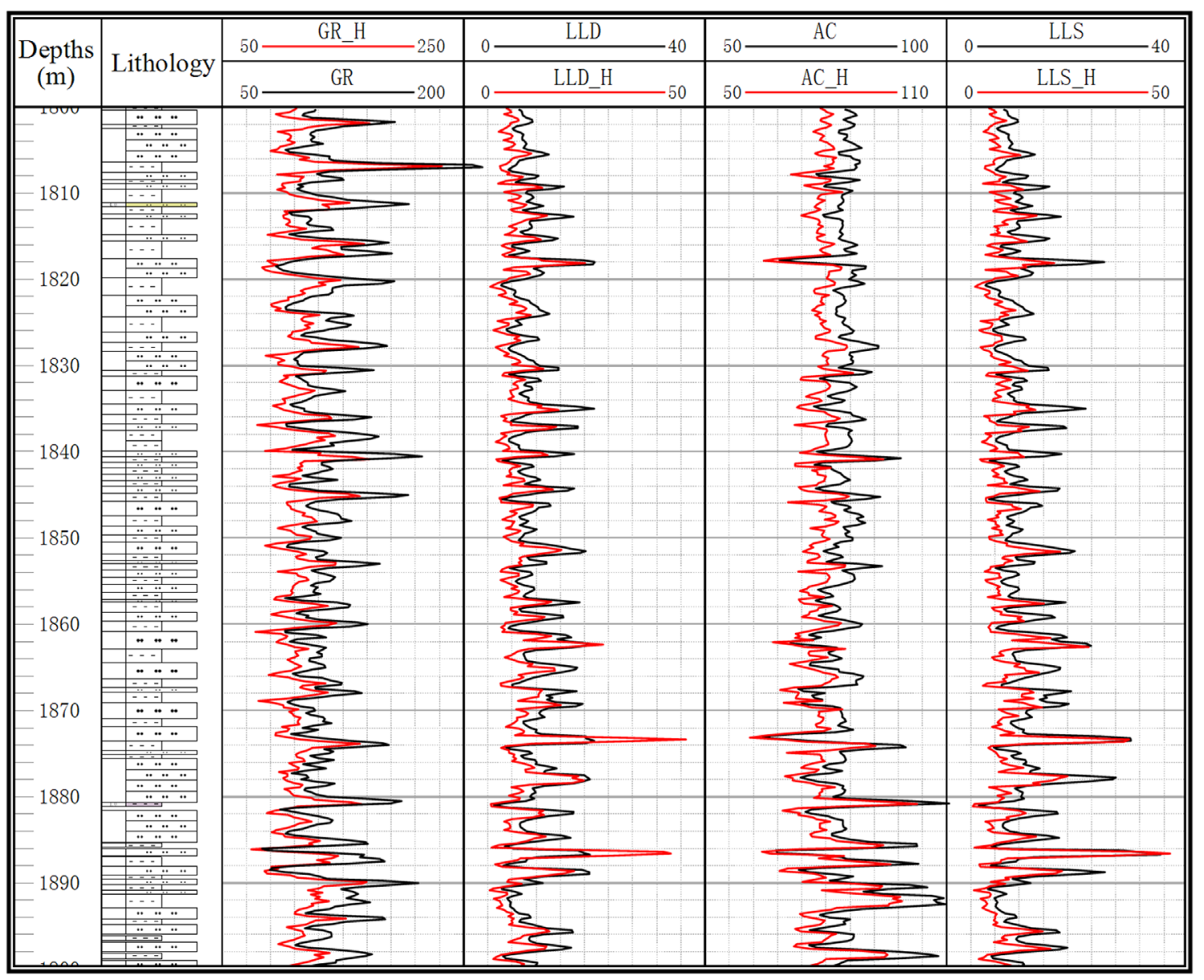

Fig. 7. Comprehensive interpretation diagram of Well_3 logging curve.

result obtained by BSpInterp method looks pretty good, but there are two obvious shortcomings: (1) high frequency information gain is very small; and (2) the problem of peak shift. Compared with the above three methods, the proposed method is relatively stable and rich in preserving the overall contour and details of the curve. Obvious, the intrinsic local structure of the log data can be successfully reconstructed by using the LLE technique. In order to better reflect this result, taking GR log data as example, power spectrums of these methods were calculated and quantitative comparisons with the power spectrum of the original high-resolution GR log data were calculated. Specifically, the corresponding power spectrums were illustrated in Figure 8. Then, each power spectrum was divided into three subbands, namely the low frequency band $(1 \mathrm{~Hz}-100 \mathrm{~Hz})$, the medium frequency band $(100 \mathrm{~Hz}-300 \mathrm{~Hz})$, and the high frequency band $(300 \mathrm{~Hz}-$ $500 \mathrm{~Hz}$ ). The Pearson coefficient values between superresolution results of different methods and the original high-resolution GR log data were given in Table 6. From Table 6 , we can clearly see that the proposed method can recover more high frequency components than other comparison methods, and the recovered low frequency and medium frequency bands are also excellent. Quantitative evaluation results of different test manners given in Tables 2 and 3 can also verify that the proposed method can achieve the best super-resolution results. In addition, we can see that unavoidable error propagation in the indirect $4 X$ super-resolution manner makes it worse than the $2 X$ and direct $4 X$ manners. And $4 X$ super-resolution results are worse than the results achieve in the $2 X$ manner because of the original information loss existing in the $1 / 4$ scale lowresolution data. Obviously, error propagation will lead to degradation of super-resolution performance. Furthermore, we can see from Table 4 that the proposed LLE-based well log super-resolution method is the fastest one among all the employed super-resolution methods. In experiment shown in Section 4.4, the proposed method is also the best one with stable and robust super-resolution performance. For the shortage of the proposed method, especially compared with BSpInterp method, the requirement of high-resolution model data limits its application value. Fortunately, for a given target exploration area, a variety of high-resolution well logs are commonly collected in some reference wells. Therefore, the shortage of the proposed method can be greatly alleviated. 


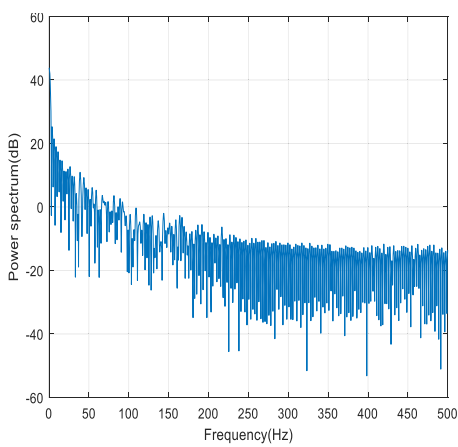

(a)

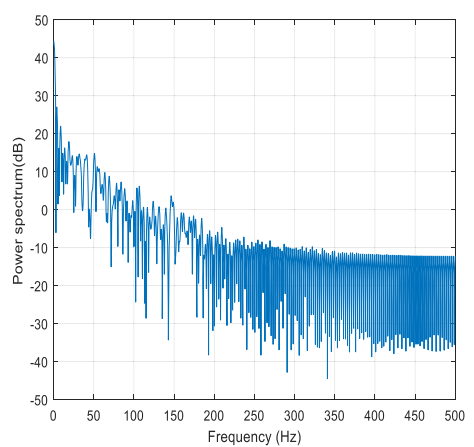

(b)

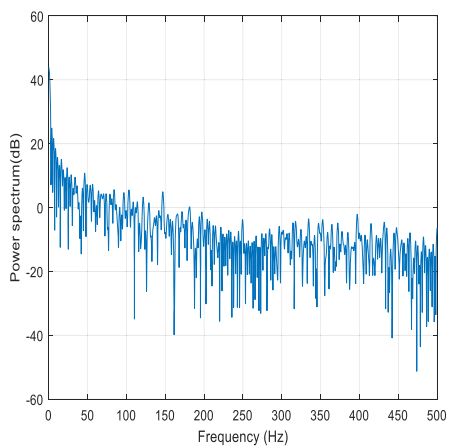

(c)

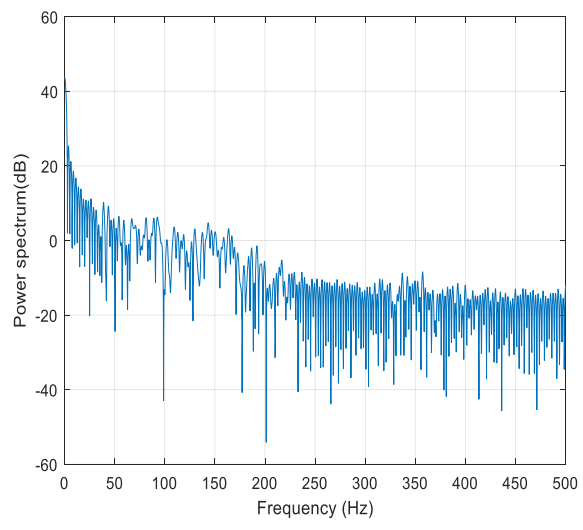

(d)

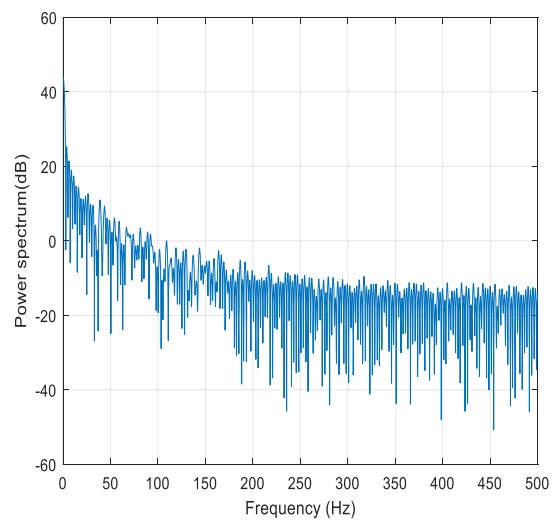

(e)

Fig. 8. Illustration of the power spectrum comparison of different methods: a) Original GR; b) Bicubic Interpolation; c) Sparse representation; d) SRCNN; e) LLE.

Table 6. Comparison of the correlation coefficients of different sub-bands of the power spectrums between of original high-resolution data and the super-resolution ones of different methods.

\begin{tabular}{lccc}
\hline Correlation & Low frequency band & Medium frequency band & High frequency band \\
\hline LLE & 0.9995 & 0.9541 & 0.9343 \\
Interpolation & 0.9994 & 0.9909 & 0.9288 \\
Sparse representation & 0.9344 & 0.7139 & 0.7149 \\
SRCNN & 0.6969 & 0.2886 & 0.2315 \\
\hline
\end{tabular}

\section{Conclusion}

In this paper, a novel well log super-resolution technique was proposed. In order to preserve or recovery as much the detailed structure information as possible during the well log super-resolution process, LLE technique was introduced for the super-resolution task. From the experimental results we can see that commonly three kinds of problems occurred for other comparison methods: (1) detailed structure information cannot be recovered satisfactorily; (2) obvious peak shift problem occurred; (3) the error fluctuation cannot be handled well. For the proposed LLE-based super-resolution method, its performance in experiments conducted with all the three test manners described in Section 4.3 is promising. Furthermore, the robustness of the proposed method is also verified in Section 4.4, which shows stable super-resolution results for well log data from different test wells. In general, the results given in this paper illustrate that LLE technique can achieve stable and reliable super-resolution of well log data.

\section{References}

1 Zou C., Yang Z. (2019) Establishment and practice of unconventional oil and gas geology, Acta Geol. Sin. 93, 01, $12-23$. 
$2 \mathrm{Su}$ J.L., Zhao Y., He T., Luo P.Y. (2021) Prediction of drilling leakage locations based on optimized neural networks and the standard random forest method, Oil Gas Sci. Technol.-Rev. IFP Energies nouvelles 76, 24.

3 Deng Y., Guo R., Tian Z.Y., Zhao L.M., Hu D.D., Liu H.Y., Liu Y. (2020) Water saturation modeling using modified J-function constrained by rock typing method in bioclastic limestone, Oil Gas Sci. Technol.-Rev. IFP Energies nouvelles 75, 66.

4 Shafiabadi M., Kamkar-Rouhani A., Sajadi S.M. (2021) Identification of the fractures of carbonate reservoirs and determination of their dips from FMI image logs using Hough transform algorithm, Oil Gas Sci. Technol.-Rev. IFP Energies nouvelles $\mathbf{7 6}, 37$.

5 Bourbiaux B. (2010) Fractured reservoir simulation: a challenging and rewarding issue, Oil Gas Sci. Technol.-Rev. IFP Energies nouvelles 65, 2, 227-238.

6 Flaum C., Galford J.E., Hasting A. (1989) Enhanced vertical resolution processing of dual detector gamma-gamma density logs, The Log Analyst 29, 5, 6, 150-157.

7 Nelson R.J., Mitchell W.K. (1992) Improved vertical resolution of well logs by resolution matching, The Log Analyst 32, 4, 281-301.

8 Liu Y.M., Zou C.C. (2006) A new method of high-resolution processing of well Logs based on genetic algorithm, Prog. Geophys. 21, 4, 1202-1207.

9 Conaway J.G. (1980) Exact inverse filters for the deconvolution of gamma-ray logs, Geoexplorations 18, 1-14.

10 Freedman R., Minerbo G.N. (June 1991) Maximum entropy inversion of induction-log data, SPE Form. Eval. 6, 02, 183-200.

11 Tai Z.W., Cao S.M. (2005) Application of Walsh transform in improving logging curve resolution, J. Shengli Oilfield Staff Univ. 04, 47-48.

12 Li X., Zhang Z.H., Su H.C. (2015) Improving the resolution of logging curve based on window Fourier transform, Mod. Chem. Indus. 44, 06, 1406-1407.
13 Lu G.Y., Wong D.W. (2007) An adaptive inverse-distance weighting spatial interpolation technique, Comput. Geosci. 34, 9, 1044-1055.

14 Dong C., Loy C.C., He K., Tang X. (2016) Image superresolution using deep convolutional networks, IEEE Trans. Pattern Anal. Mach. Intell. 38, 2, 295-307.

15 Yang J., Wright J., Huang T., Ma Y. (2010) Image superresolution via sparse representation, Image Process. IEEE Trans. 19, 11, 2861-2873.

16 Aharon M., Elad M., Bruckstein A. (2006) K-SVD: an algorithm for designing over complete dictionaries for sparse representation, IEEE Trans. Sig. Proc. 54, 11, 4311-4322.

17 Jiang B. (2015) Method of improving acoustic well logging curve resolution based on compressed sensing, Petroleum Pipes Instrum. 1, 05, 34-36+41.

18 Ledig C., Theis L., Huszar F., Caballero J., Cunningham A., Acosta A., Aitken A., Tejani A., Totz J., Wang Z. (2017) Photo-realistic single image super-resolution using a generative adversarial network, IEEE Computer Soc. 2017, 105-114.

19 Volodymyr K., Zayd E.S., Stefano E. (2017) Audio super resolution using neural networks.

20 Lim T.Y., Yeh R.A., Xu Y., Do M.N., Hasegawa-Johnson M. (2018) Time-frequency networks for audio super-resolution, in: ICASSP 2018 - 2018 IEEE International Conference on Acoustics, Speech and Signal Processing (ICASSP). IEEE.

21 Chang H., Yeung D.Y., Xiong Y. (2004) Super-resolution through neighbor embedding, in: IEEE Computer Society Conference on Computer Vision $\&$ Pattern Recognition, IEEE.

22 Zhang D.P., Fan C.K., Kuang D.Q. (2019) Impact assessment of interlayers on geological storage of carbon dioxide in Songliao Basin, Oil Gas Sci. Technol.-Rev. IFP Energies nouvelles $\mathbf{7 4}, 85$. 